\title{
Supporting Information. Cubic-Plus-Chain III: Modeling Polymer- Solvent Phase Behavior with the Chain-Modified Cubic Equation of State
}

Appendix. PC-SAFT Simulation Parameters and Polymer Molecular Weight Distributions

Table S1. PC-SAFT simulation parameters for selected solvents. ${ }^{12}$

\begin{tabular}{lcccc}
\hline \multicolumn{1}{c}{ component } & $M_{w}[\mathrm{~g} / \mathrm{mol}]$ & $\sigma_{j}[\AA]$ & $\epsilon_{j} / k[\mathrm{~K}]$ & $m_{j}[-]$ \\
\hline ethylene & 28.054 & 3.4450 & 176.47 & 1.5930 \\
propane & 44.096 & 3.6184 & 208.11 & 2.0020 \\
n-pentane & 72.149 & 3.7729 & 231.20 & 2.6896 \\
toluene & 92.138 & 3.7169 & 285.69 & 2.8149 \\
ethylbenzene & 106.165 & 3.7974 & 287.35 & 3.0799 \\
\hline
\end{tabular}

Table S2. PC-SAFT simulation parameters for selected polymers. ${ }^{13}$

\begin{tabular}{lccc}
\hline \multicolumn{1}{c}{ component } & $m / M_{w}[\mathrm{~mol} / \mathrm{g}]$ & $\sigma_{j}[\AA]$ & $\epsilon_{j} / k[\mathrm{~K}]$ \\
\hline polypropylene & 0.02305 & 4.1000 & 217.0 \\
HDPE & 0.02630 & 4.0217 & 252.0 \\
LDPE & 0.02630 & 4.0217 & 249.5 \\
polystyrene & 0.01900 & 4.1071 & 267.0 \\
\hline
\end{tabular}

Table S3. Molecular weight distribution of three pseudo-components of PP. ${ }^{22}$

\begin{tabular}{ccc}
\hline pseudo-component & $M_{w}[\mathrm{~kg} / \mathrm{mol}]$ & wt frac, $w_{p j}$ \\
\hline 1 & 35.76 & 0.5084376 \\
2 & 504.68 & 0.4792906 \\
3 & 2438.72 & 0.01227183 \\
\hline
\end{tabular}


Table S4. Molecular weight distribution of six pseudo-components of LDPE. ${ }^{24}$

\begin{tabular}{ccc}
\hline pseudo-component & $M_{w}[\mathrm{~kg} / \mathrm{mol}]$ & wt frac, $w_{p j}$ \\
\hline 1 & 2.57 & 0.1136765 \\
2 & 76.20 & 0.4774815 \\
3 & 244.00 & 0.317655 \\
4 & 513.00 & 0.0821108 \\
5 & 899.00 & 0.0087705 \\
6 & 1450.00 & 0.0003057 \\
\hline
\end{tabular}

